\title{
Atomic-Scale Deformations at the Interface of a Mixed-Dimensional van der Waals Heterostructure
}

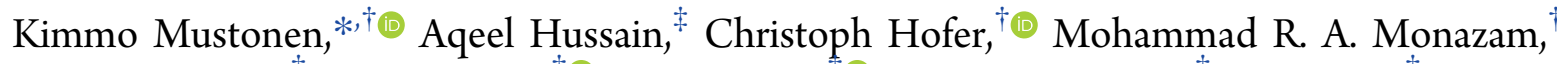
Rasim Mirzayev, ${ }^{\dagger}$ Kenan Elibol, ${ }^{\dagger}{ }^{\circ}$ Patrik Laiho, ${ }^{+\odot}$ Clemens Mangler, ${ }^{\dagger}$ Hua Jiang, ${ }^{\ddagger}$ Toma Susi, ${ }^{\dagger}$ Esko I. Kauppinen, Jani Kotakoski, ${ }^{\dagger}$ and Jannik C. Meyer* ${ }^{*}{ }^{\dagger}$

${ }^{\dagger}$ University of Vienna, Faculty of Physics, 1090 Vienna, Austria

${ }^{\ddagger}$ Aalto University School of Science, Department of Applied Physics, P.O. Box 15100, FI-00076 Aalto, Finland

Supporting Information

ABSTRACT: Molecular self-assembly due to chemical interactions is the basis of bottom-up nanofabrication, whereas weaker intermolecular forces dominate on the scale of macromolecules. Recent advances in synthesis and characterization have brought increasing attention to twoand mixed-dimensional heterostructures, and it has been recognized that van der Waals (vdW) forces within the structure may have a significant impact on their morphology. Here, we suspend single-walled carbon

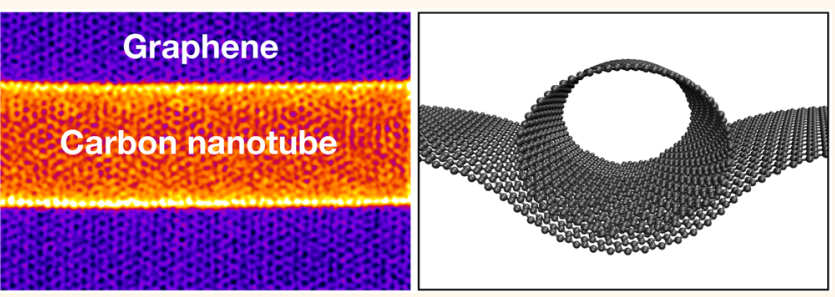
nanotubes (SWCNTs) on graphene to create a model system for the study of a 1D-2D molecular interface through atomic-resolution scanning transmission electron microscopy observations. When brought into contact, the radial deformation of SWCNTs and the emergence of long-range linear grooves in graphene revealed by the three-dimensional reconstruction of the heterostructure are observed. These topographic features are strain-correlated but show no sensitivity to carbon nanotube helicity, electronic structure, or stacking order. Finally, despite the random deposition of the nanotubes, we show that the competition between strain and vdW forces results in aligned carbon-carbon interfaces spanning hundreds of nanometers.

KEYWORDS: carbon nanotube, graphene, scanning transmission electron microscopy, elasticity, interfacing

$\mathrm{B}$ uilding on enormous advances in graphene research, interest has recently shifted to the creation of so-called van der Waals heterostructures (vdWHs). These typically combine graphene and other two- ${ }^{1}$ or lower-dimensional $^{2,3}$ molecules into vertical stacks. The principal idea of this concept is to preserve the covalent bonding structure of the molecules that interact with each other mainly through van der Waals (vdW) forces while the electronic and plasmonic coupling between the layers remains relatively strong and enables the creation of functional (meta)materials. ${ }^{1,3-7}$ However, regardless of the nominally weak vdW interaction, the lattice mismatches in these structures can result in significant morphological changes. 8,9

The creation of exclusively $2 \mathrm{D}$ heterostructures has proven relatively straightforward, either by directly placing two sheets into contact ${ }^{1}$ or via epitaxial growth. ${ }^{10-14}$ Furthermore, the energetic tendency to maximize the contact area seems to drive self-cleaning within the vdW gap, ${ }^{15}$ enabling atomically pure interfaces. However, it is much more challenging to obtain sufficiently clean, ordered, and thin layers of lower-dimensional structures owing to their higher chemical reactivity. This in turn has hindered the efforts to experimentally study fundamental phenomena governing the interactions between the layers. We recently studied monolayers of $\mathrm{C}_{60}$ molecules trapped in between two graphene sheets ${ }^{16}$ in a higher-dimensional analogue of carbon pea pods. ${ }^{17}$ In this mixed-dimensional heterostructure, we observed the deformation of the graphene sheets only at the edges of $\mathrm{C}_{60}$ molecular layers.

Here, to create a $1 \mathrm{D}-2 \mathrm{D}$ molecular interface, we have drydeposited single-walled carbon nanotubes (SWCNTs) on suspended monolayer graphene. Using scanning transmission electron microscopy (STEM), we study how they stack onto graphene and how the adsorbtion changes the morphology of both materials. Unlike most other molecules of this class, SWCNTs are hollow tubes that may deform when interacting with substrates, ${ }^{18-20}$ and although their covalent structure remains intact, this is expected to affect their transport

Received: May 29, 2018

Accepted: July 17, 2018

Published: July 17, 2018 

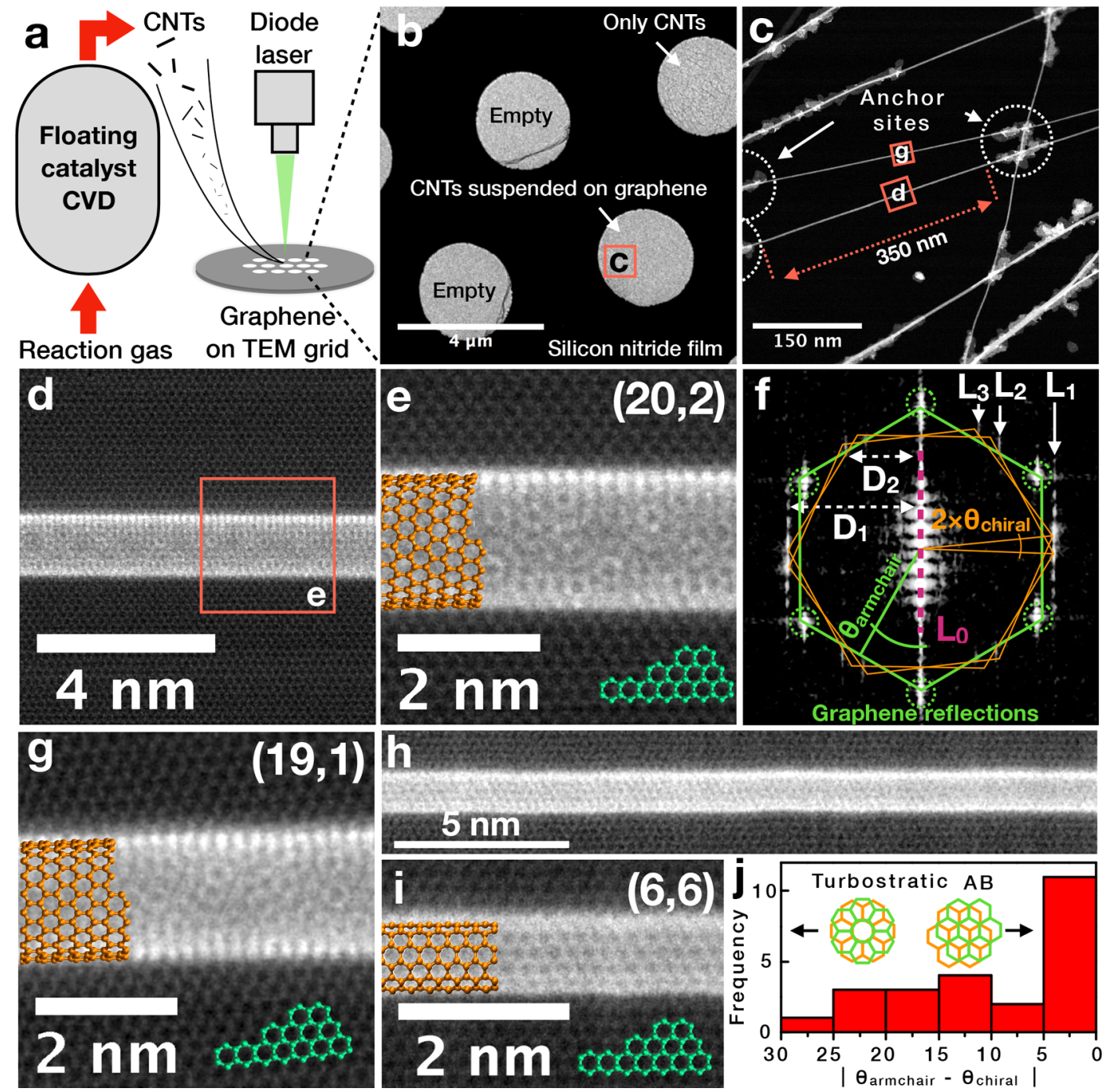

Graphenëreflections

Figure 1. SWCNT-graphene interface. (a) Heterostructure fabrication by the thermophoretic deposition ${ }^{29}$ of floating catalyst CVD SWCNTs ${ }^{27,28}$ on graphene and laser cleaning in vacuum. ${ }^{30}$ (b) Bright-field overview of a typical laser-cleaned sample (tubes on graphene cannot be seen at this magnification) and (c) a STEM MAADF image from the region marked as [c]. (d, e) Atomic-resolution close-up of a tube in region $[\mathrm{d}]$ in contact with graphene and (f) corresponding Fourier transform (FT). The chiral indices analyzed from the FT correspond to $(20,2)$. $(\mathrm{g})$ Close-up of region $[\mathrm{g}]$ showing a $(19,1)$ tube and $(\mathrm{h}, \mathrm{i})$ a $(6,6)$ armchair tube completely aligned by $A B$ stacking with graphene, i.e., $\left|\theta_{\text {armchair }}-\theta_{\text {chiral }}\right|=0^{\circ}$. (j) Histogram of observed stacking angles for 24 distinct interfaces, with $30^{\circ}$ representing turbostratic and $0^{\circ}$ representing $\mathrm{AB}$ stacking.

properties. $^{21}$ Likewise, the relative orientation strongly influences their electronic coupling: in earlier experiments, the contact barriers between graphite and carbon nanotubes were modulated by an order of magnitude simply by changing the orientation. $^{22}$

Through three-dimensional reconstructions of the interfaces, we find that graphene partially folds around the suspended molecules. The interplay between vdW and elastic energies allows them to maximize the contact area and thus minimize the total energy of the system. Much like a canvas strained by a mass that it supports, the graphene membrane also becomes slightly strained. This tension dampens the mechanical vibrations of clean suspended areas, allowing the vdWHs to be imaged at resolutions higher than separately possible for either material as cleanly as here. Unlike their vacuum-suspended counterparts, individual atoms of the tubes embedded in heterostructures can be resolved not just near the suspension point ${ }^{23}$ but also over their entire length. Complementary to scanning probe techniques, ${ }^{24,25}$ this approach may prove useful for the direct identification of point defects at ambient temperature.

Finally, we find that the carbon nanotubes align on the surface according to the symmetry of graphene, presumably to optimize the stacking of their lower wall with the underlying lattice. Nanotubes have been previously observed to grow epitaxially on graphite step edges, ${ }^{26}$ but here they self-order on ultraclean graphene due to thermal excitation.

\section{RESULTS AND DISCUSSION}

The heterostructures were fabricated as in Figure 1a, starting with the synthesis of carbon nanotubes by floating catalyst chemical vapor deposition $(\mathrm{CVD})^{27,28}$ followed by direct deposition $^{29}$ onto monolayer graphene and cleaning by laser irradiation in vacuum. ${ }^{30}$ The cleaning procedure exposed large atomically pure areas of up to $1 \mu \mathrm{m}^{2}$ in size for atomic-resolution scanning transmission electron microscopy (STEM) analysis, as shown by the medium-angle annular dark field (MAADF) image 


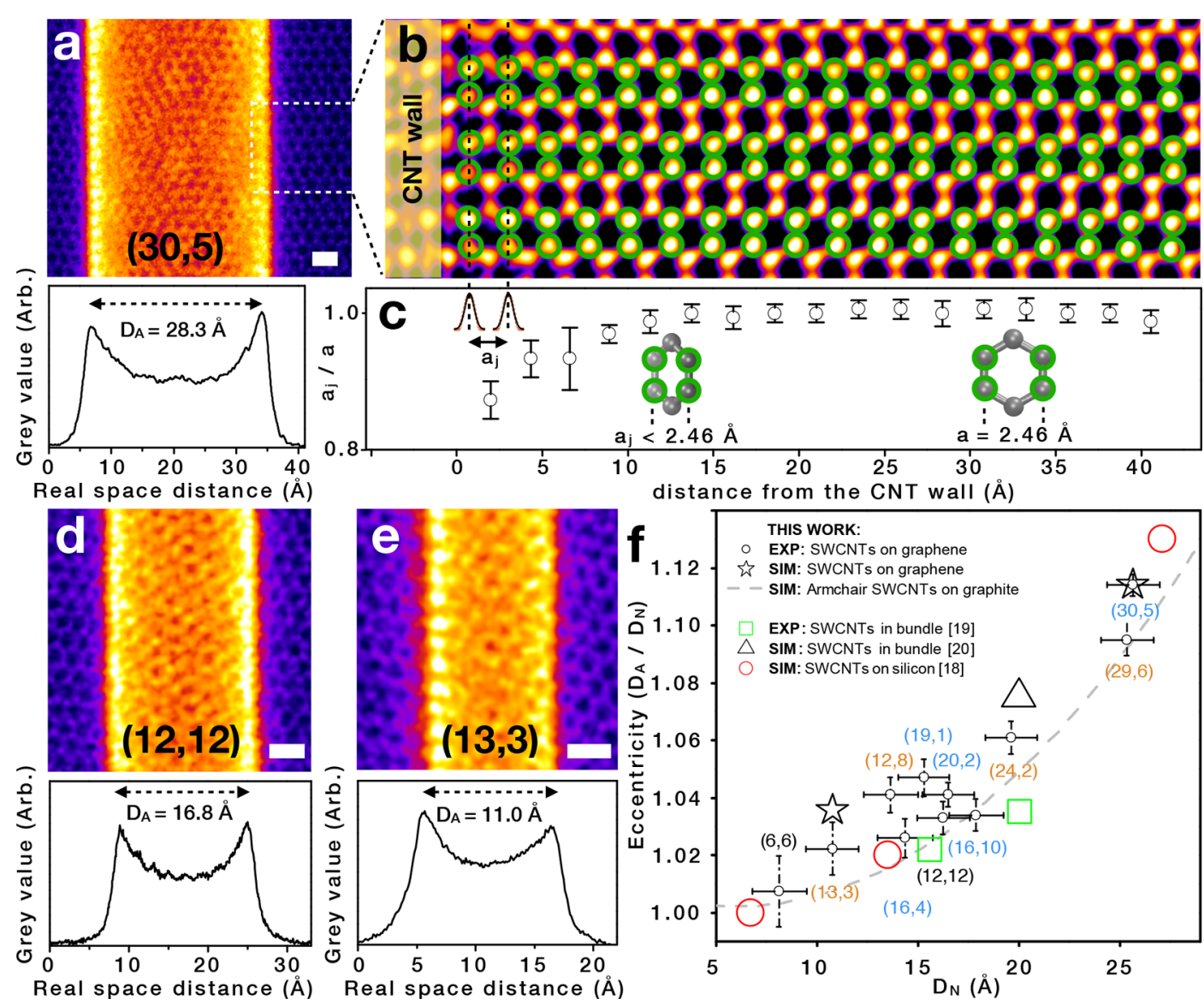

Figure 2. SWCNT deformation. (a) Large-diameter $(30,5)$ SWCNT on graphene, with a line profile revealing its apparent diameter. (b) Fourier-filtered graphene lattice next to the $(30,5)$ tube with $(c)$ the corresponding interatomic distances $a_{j}$ as a function of the distance from the SWCNT wall. (d, e) Close-ups of $(12,12)$ and $(13,3)$ tubes with their line profiles. (f) Measured eccentricity $\left(\epsilon=D_{\mathrm{A}} / D_{\mathrm{N}}\right)$ of SWCNTs suspended on graphene. The coloring of the tabulated $(n, m)$ values encodes semiconducting (orange), metallic (black), and semimetallic (blue) carbon nanotube species. Each image scale bar in $(a)$ and $(d-e)$ has a length of $0.4 \mathrm{~nm}$. To enhance the image contrast in $(a, b)$ and $(d, e)$, ImageJ lookup table "Fire" was applied.

in Figure 1c magnifying a small part of what was imaged in bright-field mode in Figure 1b. Within the example field of view, we find several SWCNTs in direct contact with graphene over hundreds of nanometers, as evidenced by their common focus. (To appear sharp, atoms need to lie within the depth of field of our STEM system, approximately $2-3 \mathrm{~nm}$, and at these distances, the molecules are brought together by vdW forces.) Unlike noncleaned samples (Supporting Information Section 1 ), these structures are devoid of contamination, hindering the view during STEM observations, and their atomic structure can be directly determined. The interfaces assume either wellaligned or meandering configurations, both visible in our examples. In the following analysis, however, we restrict ourselves to the aligned segments that exhibit the highest possible periodicity and thus structural unambiguity.

The structure of a SWCNT is completely described by its chiral indices $(n, m)$. Together with the graphene basis vectors, these span chiral vector $C_{\mathrm{h}}{ }^{31}$ Any tube can thus be constructed by wrapping a sheet of graphene around a cylinder at a chiral angle $\theta_{\text {chiral }}$ and connecting the perimeters. The chiral indices of a nanotube can be directly determined from its electron diffraction pattern, ${ }^{32}$ an atomically resolved real-space image, ${ }^{33}$ or its Fourier transform (FT). Here we exclusively use the latter approach, acquiring atomically resolved images such as those shown in Figure 1d,e,g,i. When SWCNTs are deposited on graphene, the contrast of the lattices is intermixed and structural identification requires working in Fourier space. For example, the FT of the heterostructure of Figure 1d is presented in Figure 1f, showing distinct contributions from both materials. The layer lines $L_{1 \cdots n}$ and the equatorial line $L_{0}$ arise from the lattice of the nanotube, and the graphene reflections arise from the atoms in the background. The intensity oscillations along $L_{1 \cdots n}$ and their spacing $d_{1 \cdots n}$ are mathematically related to the structure of the tube, with each line being described by a single Bessel function. ${ }^{34}$ Given that SWCNTs may compress radially on the surface and thus have an ill-defined diameter, ${ }^{18}$ the best structural unambiguity is achieved by separately analyzing the layer line spacing $^{35}$ and the oscillations along the lines ${ }^{36}$ and comparing the experiments to STEM simulations. ${ }^{37}$ For example, the best structural match for the tube in Figure 1 d,e is $(20,2)$ having a chiral angle of $4.7^{\circ}$ and nominal diameter $D_{N}$ of $16.51 \AA$. The methods for structural determination are discussed in Supporting Information Section 2.

Following this procedure, we can acquire the structure of any suspended SWCNT imaged with a sufficiently high signal-tonoise ratio. For example, the tube in Figure $1 \mathrm{~g}$ is $(19,1)$ and thus has a slightly smaller diameter of $15.28 \AA$, as also readily visible from the real-space image. A particularly interesting case of a $(6$, 6) armchair tube with an extremely small diameter of just $8.18 \AA$ appearing to be perfectly aligned with the supporting graphene 

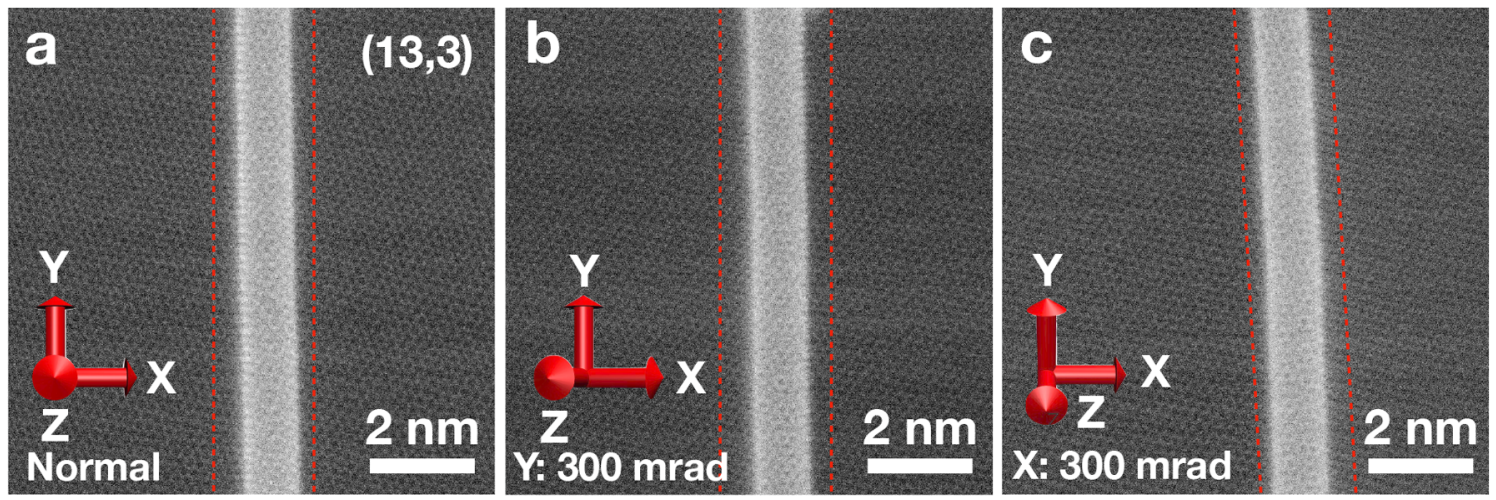

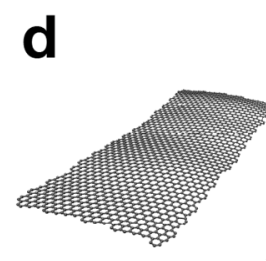

$\mathbf{f}$

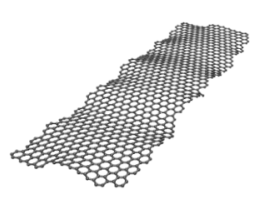

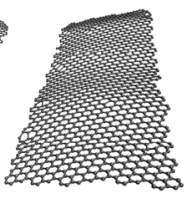

e

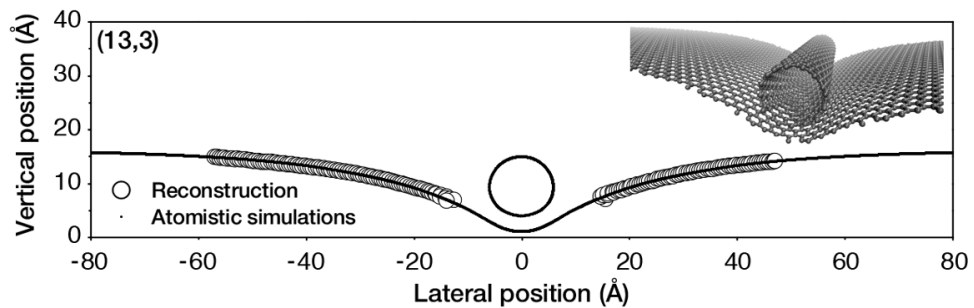

$\mathbf{g}$

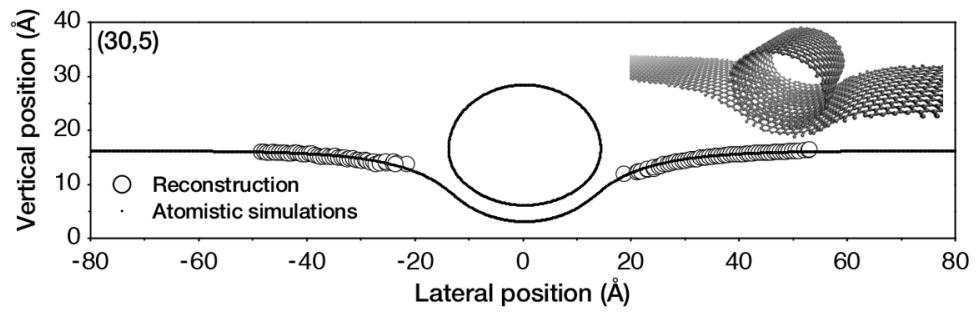

Figure 3. Graphene deformation. (a) Atomically resolved normal projection of the $(13,3)$ tube on graphene. Projections of the same site rotated by $300 \mathrm{mrad}$ around either the $y(\mathrm{~b})$ or $x$ axis $(\mathrm{c})$. (d) Reconstructed graphene lattice around the $(13,3)$ tube and the cross-section of the fitted atomistic simulations in (e). The simulated model is shown in the upper right corner as an inset. (f) Reconstruction of the graphene lattice around the $(30,5)$ tube and $(\mathrm{g})$ the fitted simulation. The experimental points in $(\mathrm{e})$ and $(\mathrm{g})$ are average atom positions perpendicular to the tube axis.

lattice is presented in Figure 1h,i. Since FT represents the frequency components of a real-space image in all directions, it also encodes the orientation of any periodic structure. Hence, we can find the relative orientation of graphene and SWCNT hexagons (although we cannot know which frequency components arise from the bottom and which arise from the top layer) by determining angles $\theta_{\text {armchair }}$ and $\theta_{\text {chiral }}$ in Fourier space (Figure 1f). For example, the $(20,2)$ tube has a helicity of $4.7^{\circ}$ (i.e., the armchair edge is rotated by this amount with respect to the tube axis), and from the FT, we see that $\theta_{\text {armchair }}$ (angle between the tube axis and the nearest graphene armchair edge) is exactly $30^{\circ}$. This gives us a misalignment angle of $25.3^{\circ}$ for the $(20,2)$ tube and $0^{\circ}$ for the $(6,6)$ tube.

We systematically analyzed 24 heterostructures for which the related angles could be reliably determined. The distribution of these angles is shown in Figure 1j. Interestingly, we observe a nonuniform distribution favoring the aligned configuration, implying that the SWCNTs are not randomly oriented despite having landed on the substrate randomly. ${ }^{29}$

We suspect that this alignment is not spontaneous but is enabled by the laser treatment, simultaneously providing the required thermal energy while removing the contamination that would hinder the movement. Approximately $55 \%$ of the heterostructures remain disordered, which could result from residual contamination stuck in the nanotube junctions, such as those presented in Figure 1c. The nanotubes appear to reach a (constrained) energy minimum within the potential landscape of graphene and hence preserve their orientation over relatively long distances. Interestingly, the aligned structures not only have the highest possible symmetry but also allow the nanotube handedness to be directly extracted based on the graphene orientation. $^{26}$

We now turn our attention to the detailed response of the heterostructure to dispersion forces, first concentrating on the nanotubes. After seminal molecular mechanics predictions in the early $1990 \mathrm{~s}^{20}$ the radial deformation of SWCNTs as influenced by substrates has also been studied experimentally. ${ }^{18,19}$ Most investigations, however, have been limited to scanning probe methods, ${ }^{18,38,39}$ posing problems with tip deconvolution, the vdW distance to the substrate, and surface roughness. ${ }^{38}$ Moreover, a structural determination using these techniques is extremely demanding. ${ }^{25}$ In contrast, our heterostructures provide the first clean view to measure such effects using an electron probe free of these confounding factors. Furthermore, the image scale can be directly calibrated in Fourier space by using the nearest-neighbor distance in graphene and finally the apparent diameter $\left(D_{\mathrm{A}}\right)$ measured at sub-angstrom accuracy.

The eccentricity $(\epsilon)$ of a deformed tubular object can be described as the ratio of the major and minor axes of the ellipse it forms. Here we use a slightly different definition, $\epsilon=D_{\mathrm{A}} / D_{\mathrm{N}}$, i.e., comparing the apparent and nominal diameters directly. For this comparison, we have chosen three tubes $(13,3),(12,12)$, and $(30,5)$ with nominal diameters of $10.79,16.29$, and $25.67 \AA$, 

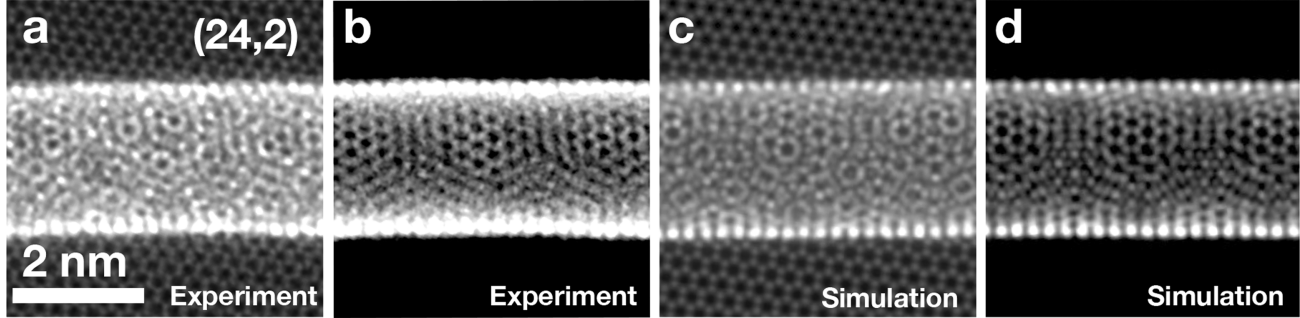

Figure 4. Imaging stability. (a) A STEM MAADF image of a $(24,2)$ SWCNT supported on graphene and (b) the same image after Fourier filtering the graphene. (c) Image simulation of the same structure with graphene removed in (d). Note that the signal-to-noise ratio in (a, b) was improved by double Gaussian filtering. ${ }^{42}$

respectively, as shown in Figure 2a,d,e. To determine their eccentricity, we analyzed the wall separation by fitting Gaussian line shapes to the nanotube cross-sectional intensity profiles shown below the real-space images. For example, the smalldiameter $(13,3)$ tube is quite rigid, undergoing only a small deformation to $\epsilon=1.022$. The slightly larger $(12,12)$ tube flattens more to an eccentricity of 1.033 , and the $(30,5)$ tube flattens to as high as 1.114. In total, we studied the deformation of 10 structurally identified tubes, with the results gathered in Figure 2f. Neither the chiral angle nor the electronic type (semiconductor or metal) appear to be correlated with the amount of deformation, which confirms earlier theoretical predictions. $^{40}$ Generally speaking, however, and especially considering the accuracy of our method, the data shows a large variation in $\epsilon$. Surprisingly, deformation on graphene seems to be greater than what is separately reported for bundled SWCNTs ${ }^{19}$ or on bulk substrates. ${ }^{18}$

To understand these discrepancies, we used atomistic simulations (Methods) to study the adsorption of SWCNTs on both graphene and graphite. Graphite was imitated by completely fixing a single layer of graphene, whereas to reproduce the curvature of graphene found in experiments (discussed next), the simulation cell perpendicular to tube axis was initially compressed by $0.7-0.8 \%$, leaving the graphene under a negative strain and thus free to adapt to the adsorbed tube. Simulations were performed on (i) $(13,3)$ and $(30,5)$ tubes suspended on graphene and marked with star symbols in Figure $2 \mathrm{f}$ and (ii) for a number of armchair tubes suspended on graphite, as indicated by the dashed line.

Interestingly, tubes on graphene show larger deformations than on graphite, $\epsilon=1.038$ vs 1.009 for $(13,3)$ and 1.110 vs 1.090 for $(30,5)$. This initially counterintuitive result is explained by an increase in binding energy on graphene, in respective order yielding 0.085 vs $0.176 \mathrm{eV} / \AA ̊$ and 0.205 vs 0.411 $\mathrm{eV} / \AA$. . This is a direct consequence of graphene minimizing the surface energy by partially folding around the nanotube as in Figure $3 \mathrm{e}-\mathrm{g}$ and Supporting Information Figure S5. Thus, the gain in surface energy is achieved by the greater structural deformation of the tube.

The observed variation in $\epsilon$ can be similarly explained: randomly oriented tubes on isotropically strained graphene undergo minimum deformation, but a uniaxial strain allows graphene to bend perpendicular to this axis, with some of the tubes deviating from the curve.

Apparently, the real-world membrane behaves like this, with the strain continuously varying in both direction and magnitude at different locations on the sample.

We turn our attention next to graphene, which ideally is a perfectly two-dimensional crystal. We start by analyzing the interatomic spacing of the graphene lattice around the carbon nanotubes. In normal projection, a planar topology has isotropic atomic separations quantized to $60^{\circ}$ angles. This, however, systematically changes on a curved surface, as schematically depicted in the lower part of Figure $2 \mathrm{c}$. Here, we chose the lattice near the $(30,5)$ tube and used Fourier filtering to produce a clearer view of the atom positions, shown in Figure $2 \mathrm{~b}$. From the filtered image, we extracted the interatom separations by fitting Gaussian line shapes to the intensity profiles of each atom, highlighted by the green circles, producing the data shown in Figure $2 c$. If we schematically approach the interface from the right-hand side, then we observe a decrease in the apparent atom separation, extending $\sim 20 \AA$ from the edge and thus implying local out-of-plane curvature. By this method alone, however, the vertical direction of the inclination is ambiguous.

This limitation can be overcome by analyzing atomicresolution images of the sample recorded from the same area under different tilt angles. We have recently developed an algorithm that obtains the $3 \mathrm{D}$ structure of a $2 \mathrm{D}$ material (graphene) from a minimum of two projections with a relative sample tilt of about $15-20^{\circ} .41$ The algorithm identifies the position of each atom within the experimental projections and then finds the best-matching 3D structure that minimizes the difference between simulated images of the structure and the available experimental ones. Figure $3 b, c$ shows projections of the $(13,3)$ tube on graphene acquired by rotating the sample separately around the $x$ and $y$ axes by $300 \mathrm{mrad}\left(17.2^{\circ}\right)$ relative to the normal incidence in Figure 3a. Since only the structure of the single-layer graphene can be unambiguously reconstructed, the atom positions between the red dashed lines were omitted from the analysis, finally yielding the $3 \mathrm{D}$ atomic model shown in Figure $3 \mathrm{~d}$. To recover the complete structure including the nanotube, we performed several atomistic simulations with a varying degree of strain in the graphene to simultaneously match the result to experimental graphene curvature and SWCNT eccentricity (Methods). As readily visible in Figure 3e, the simulation reveals an $\sim 14$ - $\AA$-deep $1 \mathrm{D}$ groove symmetrically expanding on both sides of the tube. The experimental points here represent the average vertical positions of atoms perpendicular to the tube axis. Following the same procedure, we also studied the graphene curvature around the $(30,5)$ tube, for which the reconstructed lattice and simulation are shown in Figure $3 f, g$, respectively.

Finally, given the high adsorption energies and the strain in graphene, these hybrid structures are extremely stable in our STEM experiments. Images can be acquired from arbitrary positions at resolution exceptional for such large and atomically clean systems. Furthermore, graphene can be effectively eliminated from the data by Fourier filtering. ${ }^{16}$ This reveals a beautiful view of the SWCNT lattice from which the positions of individual atoms can be resolved along the tube axis. Such an 
example is shown in Figure 4a,b, displaying a $(24,2)$ tube with and without the graphene background. These images are extremely sharp, exhibiting a striking resemblance to the image simulations in Figure 4c,d.

\section{CONCLUSIONS}

By using graphene and single-walled carbon nanotubes as a model heterostructure, we have studied atomic-scale deformations in a $1 \mathrm{D}-2 \mathrm{D}$ molecular interface held together by van der Waals forces. The interface shows topographically interesting features, with nanotubes dipping into the supporting graphene layer, creating ordered, long-range, one-dimensional grooves that reduce the surface energy of the structure. With the help of multiple projections, the three-dimensional structure of the lattice can be reconstructed, and when correlated with atomistic simulations, the morphology of the complete heterostructure is recovered. The graphene support thus allows the nanotubes to be observed along their entire length at room temperature with accuracy previously possible only through scanning probe techniques on rigid substrates.

\section{METHODS}

Sample Fabrication. Carbon nanotubes were synthesized using floating catalyst chemical vapor deposition. For small-diameter tubes, we used carbon monoxide as the carbon precursor, decomposing on exsitu-generated iron nanoparticles at $880{ }^{\circ} \mathrm{C} .{ }^{27}$ The larger-diameter tubes were synthesized at $1050{ }^{\circ} \mathrm{C}$ by ethylene precursor decomposing on nanoparticles formed in situ from ferrocene vapor. ${ }^{28}$ The assynthesized tubes were dry-deposited ${ }^{29}$ on commercial CVD graphene (Graphenea Inc.) suspended on perforated silicon nitride grids (Ted Pella Inc.) The structures were cleaned by $20 \mathrm{~s}$ of laser irradiation at $3 \mathrm{~W}$ excitation power ${ }^{30}$ under $10^{-7} \mathrm{~Pa}$ pressure and transferred to the microscope column within the same vacuum system.

Scanning Transmission Electron Microscopy. Atomic-resolution imaging used the aberration-corrected Nion UltraSTEM 100 operated with a $60 \mathrm{keV}$ primary beam energy, with the sample in a nearultrahigh vacuum of $2 \times 10^{-7} \mathrm{~Pa}$. The angular range for the mediumangle annular dark-field (MAADF) detector was 60-200 mrad. Sample tilt was eliminated by bringing a $64 \times 64 \mathrm{~nm}$ field of view completely into focus by rotating the sample in a standard Nion double-tilt holder. With our 2 to $3 \mathrm{~nm}$ depth of field, this corresponds to a maximum tilt of less than $5^{\circ}$, providing good unambiguity in the $(n, m)$ assignment. ${ }^{37}$ To minimize spatial inaccuracies caused by scan distortions (i.e., variations in the vertical scan speed) or drift, the nanotube diameters were measured from images acquired by setting the scan direction perpendicular to the carbon nanotube axis. For reliable $(n, m)$ assignment, both parallel and perpendicular scans were used and compared.

Scanning Transmission Electron Microscopy Simulations. STEM simulations were performed using QSTEM 2.31 with a chromatic aberration coefficient of $1 \mathrm{~mm}$, a spherical aberration coefficient of $1 \mu \mathrm{m}$, an energy spread of $0.48 \mathrm{eV}$, and MAADF detector angles set to the experimental range of 60-200 mrad. The illumination semiangle was $35 \mathrm{mrad}$. In Figure $4 \mathrm{c}$,d, the best match with the experiment was achieved by placing the carbon nanotube at a van der Waals distance $(0.34 \mathrm{~nm}$ ) from the graphene membrane (without energy optimization) and rotating the whole system by $5^{\circ}$ around the principal axis perpendicular to the tube, with the electron beam then encountering the sample plane at this angle. The chiral index, $(24,2)$, and the relative graphene orientation in the simulation were identified from the experimental image as described in the text and in Supporting Information Section 2. To mimic the experimental contrast in Figure $4 \mathrm{~d}$, graphene was first included in the simulations and manually removed by Fourier filtering.

Three-Dimensional Reconstruction. The three-dimensional reconstruction was done by matching a simulated model to a series of experimental images acquired from different tilt angles by rotating the sample to $\pm 300 \mathrm{mrad}$ inclination during imaging. Finally, the structure was obtained through an optimization process where both the atomic positions and the simulated imaging parameters were iteratively changed until the best possible match to the experiment was found. ${ }^{41}$ This was possible since the location of each graphene lattice atom and their connections to nearest neighbors could be discerned from each projection.

Atomistic Simulations. The adaptive intermolecular reactive empirical bond order (AIREBO) ${ }^{43}$ potential was used to describe the covalent bonding in graphene and nanotubes, whereas $\mathrm{vdW}$ interactions were included by augmenting the model with a Morse potential. ${ }^{44}$ The torsion component of the AIREBO potential for the nanotube was turned off since this gave a better match with the experimental results, and the Morse potential parameters were identical to the AIREBO Morse potential. ${ }^{44}$ All structural minimization processes were performed using the large-scale atomic/molecular massively parallel simulator (LAMMPS) code. ${ }^{45,46}$

For each carbon nanotube, the graphene structure was oriented so that it is commensurate with a single unit cell of the chiral nanotubes, which is necessary to enforce the periodic boundary conditions along the axis. For the $(13,3)$ and $(30,5)$ tubes, the rotated graphene unit cell was multiplied perpendicular to the axis by 12 - and 28 -fold, respectively. Hence, the total width of graphene for the $(13,3)$ tube was $\sim 64 \mathrm{~nm}$, and that for the $(30,5)$ tube was $\sim 66 \mathrm{~nm}$. To match the graphene curvature with the reconstructed results, the bounding box of the cell was shrunk by 0.7 and $0.8 \%$ for the $(13,3)$ and $(30,5)$ tubes, respectively. The nanotube eccentricity was determined by comparing the diameters of a relaxed nanotube in vacuum and on the surface.

\section{ASSOCIATED CONTENT}

\section{S Supporting Information}

The Supporting Information is available free of charge on the ACS Publications website at DOI: 10.1021/acsnano.8b04050.

Images of non-laser-treated heterostructures, description of the chiral index assignment, and the relation of adsorption energy and carbon nanotube eccentricity (PDF)

\section{AUTHOR INFORMATION}

\section{Corresponding Authors}

*E-mail: kimmo.mustonen@univie.ac.at.

*E-mail: jannik.meyer@univie.ac.at.

ORCID

Kimmo Mustonen: 0000-0002-0953-7299

Christoph Hofer: 0000-0002-0844-8366

Kenan Elibol: 0000-0002-8765-2794

Patrik Laiho: 0000-0001-8234-1607

Toma Susi: 0000-0003-2513-573X

Jannik C. Meyer: 0000-0003-4023-0778

\section{Notes}

The authors declare no competing financial interest.

\section{ACKNOWLEDGMENTS}

This work was supported by the European Research Council Starting Grant (nos. 336453-PICOMAT and 756277-ATMEN) and by the European Union Seventh Framework Programme (FP7/2007-2013) under grant agreement no. 604472-IRENA. This research has also been supported by the Austrian Science Fund (FWF) under project nos. P 25721-N20, I1283-N20, P 28322-N36, and I3181-N36, the Academy of Finland via projects 286546-DEMEC and 292600-SUPER, by TEKES Finland via projects 3303/31/2015 (CNT-PV) and 1882/31/ 2016 (FEDOC), and the Aalto Energy Efficiency (AEF) Research Program through the MOPPI project. K.M. acknowl- 
edges support from the Finnish Foundations Post Doc Pool. J.K. acknowledges funding from Wiener Wissenschafts-Forschungsund Technologiefonds through project MA14-009. Computational resources from the Vienna Scientific Cluster are gratefully acknowledged.

\section{REFERENCES}

(1) Geim, A. K.; Grigorieva, I. V. Van der Waals Heterostructures. Nature 2013, 499, 419-425.

(2) Jariwala, D.; Marks, T. J.; Hersam, M. C. Mixed-dimensional van der Waals Heterostructures. Nat. Mater. 2017, 16, 170

(3) Liu, Y.; Weiss, N. O.; Duan, X.; Cheng, H.-C.; Huang, Y.; Duan, X. Van der Waals Heterostructures and Devices. Nat. Rev. Mater. 2016, 1, 16042 .

(4) Britnell, L.; Ribeiro, R.; Eckmann, A.; Jalil, R.; Belle, B.; Mishchenko, A.; Kim, Y.-J.; Gorbachev, R.; Georgiou, T.; Morozov, S.; Grigorenko, A. N.; Geim, A. K.; Casiraghi, C.; Castro Neto, A. H.; Novoselov, K. S. Strong Light-Matter Interactions in Heterostructures of Atomically Thin Films. Science 2013, 340, 1311-1314.

(5) Zheng, Q.; Saidi, W. A.; Xie, Y.; Lan, Z.; Prezhdo, O. V.; Petek, H.; Zhao, J. Phonon-Assisted Ultrafast Charge Transfer at van der Waals Heterostructure Interface. Nano Lett. 2017, 17, 6435-6442.

(6) Gjerding, M. N.; Petersen, R.; Pedersen, T.; Mortensen, N. A.; Thygesen, K. S. Layered van der Waals Crystals with Hyperbolic Light Dispersion. Nat. Commun. 2017, 8, 320.

(7) Yu, W. J.; Vu, Q. A.; Oh, H.; Nam, H. G.; Zhou, H.; Cha, S.; Kim, J.-Y.; Carvalho, A.; Jeong, M.; Choi, H.; Castro Neto, A. H.; Lee, Y. H.; Duan, X. Unusually Efficient Photocurrent Extraction in Monolayer van der Waals Heterostructure by Tunnelling Through Discretized Barriers. Nat. Commun. 2016, 7, 13278.

(8) Argentero, G.; Mittelberger, A.; Reza Ahmadpour Monazam, M.; Cao, Y.; Pennycook, T. J.; Mangler, C.; Kramberger, C.; Kotakoski, J.; Geim, A.; Meyer, J. C. Unraveling the 3D Atomic Structure of a Suspended Graphene/hBN van der Waals Heterostructure. Nano Lett. 2017, 17, 1409-1416.

(9) Leven, I.; Maaravi, T.; Azuri, I.; Kronik, L.; Hod, O. Interlayer Potential for Graphene/h-BN Heterostructures. J. Chem. Theory Comput. 2016, 12, 2896-2905.

(10) Yang, W.; Chen, G.; Shi, Z.; Liu, C.-C.; Zhang, L.; Xie, G.; Cheng, M.; Wang, D.; Yang, R.; Shi, D.; Watanabe, K.; Taniguchi, T.; Yao, Y.; Zhang, Y.; Zhang, G. Epitaxial Growth of Single-Domain Graphene on Hexagonal Boron Nitride. Nat. Mater. 2013, 12, 792.

(11) Zhang, C.; Zhao, S.; Jin, C.; Koh, A. L.; Zhou, Y.; Xu, W.; Li, Q.; Xiong, Q.; Peng, H.; Liu, Z. Direct Growth of Large-Area Graphene and Boron Nitride Heterostructures by a Co-Segregation Method. Nat. Commun. 2015, 6, 6519.

(12) Yan, A.; Velasco, J., Jr; Kahn, S.; Watanabe, K.; Taniguchi, T.; Wang, F.; Crommie, M. F.; Zettl, A. Direct Growth of Single-and FewLayer MoS2 on h-BN with Preferred Relative Rotation Angles. Nano Lett. 2015, 15, 6324-6331.

(13) Xu, S.; Han, Y.; Chen, X.; Wu, Z.; Wang, L.; Han, T.; Ye, W.; Lu, H.; Long, G.; Wu, Y.; Lin, J.; Cai, K. M.; Yuan, Ho; He, Y.; Wang, N. van der Waals Epitaxial Growth of Atomically Thin $\mathrm{Bi}_{2} \mathrm{Se}_{3}$ and ThicknessDependent Topological Phase Transition. Nano Lett. 2015, 15, 26452651.

(14) Zuo, Z.; Xu, Z.; Zheng, R.; Khanaki, A.; Zheng, J.-G.; Liu, J. Insitu Epitaxial Growth of Graphene/h-BN van der Waals Heterostructures by Molecular Beam Epitaxy. Sci. Rep. 2015, 5, 14760.

(15) Rooney, A. P.; Kozikov, A.; Rudenko, A. N.; Prestat, E.; Hamer, M. J.; Withers, F.; Cao, Y.; Novoselov, K. S.; Katsnelson, M. I.; Gorbachev, R.; Haigh, S. J. Observing Imperfection in Atomic Interfaces for van der Waals Heterostructures. Nano Lett. 2017, 17, $5222-5228$

(16) Mirzayev, R.; Mustonen, K.; Monazam, M. R.; Mittelberger, A.; Pennycook, T. J.; Mangler, C.; Susi, T.; Kotakoski, J.; Meyer, J. C. Buckyball Sandwiches. Sci. Adv. 2017, 3, e1700176.

(17) Smith, B. W.; Monthioux, M.; Luzzi, D. E. Encapsulated $\mathrm{C}_{60}$ in Carbon Nanotubes. Nature 1998, 396, 323-324.
(18) Hertel, T.; Walkup, R. E.; Avouris, P. Deformation of Carbon Nanotubes by Surface van der Waals Forces. Phys. Rev. B: Condens. Matter Mater. Phys. 1998, 58, 13870.

(19) Jiang, Y.; Zhou, W.; Kim, T.; Huang, Y.; Zuo, J. Measurement of Radial Deformation of Single-wall Carbon Nanotubes Induced by Intertube van der Waals Forces. Phys. Rev. B: Condens. Matter Mater. Phys. 2008, 77, 153405.

(20) Ruoff, R. S.; Tersoff, J.; Lorents, D. C.; Subramoney, S.; Chan, B. Radial Deformation of Carbon Nanotubes by van der Waals Forces. Nature 1993, 364, 514-516.

(21) Kim, T.; Kim, G.; Choi, W. I.; Kwon, Y.-K.; Zuo, J.-M. Electrical Transport in Small Bundles of Single-Walled Carbon Nanotubes: Intertube Interaction and Effects of Tube Deformation. Appl. Phys. Lett. 2010, 96, 173107.

(22) Paulson, S.; Helser, A.; Nardelli, M. B.; Taylor, R.; Falvo, M.; Superfine, R.; Washburn, S. Tunable Resistance of a Carbon NanotubeGraphite Interface. Science 2000, 290, 1742-1744.

(23) Warner, J. H.; Young, N. P.; Kirkland, A. I.; Briggs, G. A. D. Resolving Strain in Carbon nanotubes at the Atomic Level. Nat. Mater. 2011, 10, 958.

(24) Ishigami, M.; Choi, H. J.; Aloni, S.; Louie, S. G.; Cohen, M. L.; Zettl, A. Identifying Defects in Nanoscale Materials. Phys. Rev. Lett. 2004, 93, 196803.

(25) Odom, T. W.; Huang, J.-L.; Kim, P.; Lieber, C. M. Atomic Structure and Electronic Properties of Single-Walled Carbon Nanotubes. Nature 1998, 391, 62-64.

(26) Chen, Y.; Shen, Z.; Xu, Z.; Hu, Y.; Xu, H.; Wang, S.; Guo, X.; Zhang, Y.; Peng, L.; Ding, F.; Liu, Z.; Zhang, J. Helicity-Dependent Single-Walled Carbon Nanotube Alignment on Graphite for Helical Angle and Handedness Recognition. Nat. Commun. 2013, 4, 2205.

(27) Mustonen, K.; Laiho, P.; Kaskela, A.; Zhu, Z.; Reynaud, O.; Houbenov, N.; Tian, Y.; Susi, T.; Jiang, H.; Nasibulin, A. G.; Kauppinen, E. I. Gas Phase Synthesis of Non-Bundled, Small Diameter Single-Walled Carbon Nanotubes With Near-Armchair Chiralities. Appl. Phys. Lett. 2015, 107, 013106.

(28) Hussain, A.; Liao, Y.; Zhang, Q.; Ding, E.-X.; Laiho, P.; Ahmad, S.; Wei, N.; Tian, Y.; Jiang, H.; Kauppinen, E. I. Floating Catalyst CVD Synthesis of Single Walled Carbon Nanotubes from Ethylene for High Performance Transparent Electrodes. Nanoscale 2018, 10, 9752-9759.

(29) Laiho, P.; Mustonen, K.; Ohno, Y.; Maruyama, S.; Kauppinen, E. I. Dry and Direct Deposition of Aerosol Synthesized Single-Walled Carbon Nanotubes by Thermophoresis. ACS Appl. Mater. Interfaces 2017, 9, 20738-20747.

(30) Tripathi, M.; Mittelberger, A.; Mustonen, K.; Mangler, C.; Kotakoski, J.; Meyer, J. C.; Susi, T. Cleaning Graphene: Comparing Heat Treatments in Air and in Vacuum. Phys. Phys. Status Solidi RRL 2017, 11, 1700124.

(31) Dresselhaus, M. S.; Dresselhaus, G.; Eklund, P.; Rao, A. The Physics of Fullerene-Based and Fullerene-Related Materials; Springer, 2000; pp 331-379.

(32) Iijima, S.; Ichihashi, T. Single-Shell Carbon Nanotubes of 1-nm Diameter. Nature 1993, 363, 603.

(33) Hashimoto, A.; Suenaga, K.; Urita, K.; Shimada, T.; Sugai, T.; Bandow, S.; Shinohara, H.; Iijima, S. Atomic Correlation Between Adjacent Graphene Layers in Double-Wall Carbon Nanotubes. Phys. Rev. Lett. 2005, 94, 045504.

(34) Amelinckx, S.; Lucas, A.; Lambin, P. Electron Diffraction and Microscopy of Nanotubes. Rep. Prog. Phys. 1999, 62, 1471.

(35) Jiang, H.; Nasibulin, A. G.; Brown, D. P.; Kauppinen, E. I. Unambiguous Atomic Structural Determination of Single-Walled Carbon Nanotubes by Electron Diffraction. Carbon 2007, 45, 662667.

(36) Liu, Z.; Qin, L.-C. A Direct Method to Determine the Chiral Indices of Carbon Nanotubes. Chem. Phys. Lett. 2005, 408, 75-79.

(37) Liu, Z.; Qin, L.-C. Electron Diffraction from Elliptical Nanotubes. Chem. Phys. Lett. 2005, 406, 106-110.

(38) Giusca, C. E.; Tison, Y.; Silva, S. R. P. Atomic and Electronic Structure in Collapsed Carbon Nanotubes Evidenced by Scanning 
Tunneling Microscopy. Phys. Rev. B: Condens. Matter Mater. Phys. 2007, 76,035429 .

(39) Barraza-Lopez, S.; Albrecht, P. M.; Lyding, J. W. Carbon Nanotubes on Partially De-Passivated n-Doped Si (100)- $(2 \times 1): \mathrm{H}$ Substrates. Phys. Rev. B: Condens. Matter Mater. Phys. 2009, 80, 045415.

(40) Li, C.; Chou, T.-W. Elastic Properties of Single-Walled Carbon Nanotubes in Transverse Directions. Phys. Rev. B: Condens. Matter Mater. Phys. 2004, 69, 073401.

(41) Hofer, C.; Kramberger, C.; Ahmadpour Monazam, M. R.; Mangler, C.; Mittelberger, A.; Argentero, G.; Kotakoski, J.; Meyer, J. C. Revealing the 3D Structure of Graphene Defects. Conference on Physics of Defects in Solids: Quantum Mechanics Meets Topology; July 12, 2018, Trieste, Italy.

(42) Krivanek, O. L.; Chisholm, M. F.; Nicolosi, V.; Pennycook, T. J.; Corbin, G. J.; Dellby, N.; Murfitt, M. F.; Own, C. S.; Szilagyi, Z. S.; Oxley, M. P.; Pantelides, S. T.; Pennycook, S. J. Atom-By-Atom Structural and Chemical Analysis by Annular Dark-Field Electron Microscopy. Nature 2010, 464, 571.

(43) Brenner, D. W. Empirical Potential for Hydrocarbons for Use in Simulating the Chemical Vapor Deposition of Diamond Films. Phys. Rev. B: Condens. Matter Mater. Phys. 1990, 42, 9458.

(44) O'Connor, T. C.; Andzelm, J.; Robbins, M. O. AIREBO-M: A Reactive Model for Hydrocarbons at Extreme Pressures. J. Chem. Phys. 2015, 142, 024903.

(45) Plimpton, S. Fast Parallel Algorithms for Short-Range Molecular Dynamics. J. Comput. Phys. 1995, 117, 1-19.

(46) Plimpton, S. J.; Thompson, A. P. Computational Aspects of Many-Body Potentials. MRS Bull. 2012, 37, 513-521. 